NASA/TM-2003-212314

\title{
A 3-D CE/SE Navier-Stokes Solver With Unstructured Hexahedral Grid for Computation of Near Field Jet Screech Noise
}

Ching Y. Loh and Ananda Himansu

Taitech, Inc., Beaver Creek, Ohio

Lennart S. Hultgren

Glenn Research Center, Cleveland, Ohio 
Since its founding, NASA has been dedicated to the advancement of aeronautics and space science. The NASA Scientific and Technical Information (STI) Program Office plays a key part in helping NASA maintain this important role.

The NASA STI Program Office is operated by Langley Research Center, the Lead Center for NASA's scientific and technical information. The NASA STI Program Office provides access to the NASA STI Database, the largest collection of aeronautical and space science STI in the world. The Program Office is also NASA's institutional mechanism for disseminating the results of its research and development activities. These results are published by NASA in the NASA STI Report Series, which includes the following report types:

- $\quad$ TECHNICAL PUBLICATION. Reports of completed research or a major significant phase of research that present the results of NASA programs and include extensive data or theoretical analysis. Includes compilations of significant scientific and technical data and information deemed to be of continuing reference value. NASA's counterpart of peerreviewed formal professional papers but has less stringent limitations on manuscript length and extent of graphic presentations.

- TECHNICAL MEMORANDUM. Scientific and technical findings that are preliminary or of specialized interest, e.g., quick release reports, working papers, and bibliographies that contain minimal annotation. Does not contain extensive analysis.

- CONTRACTOR REPORT. Scientific and technical findings by NASA-sponsored contractors and grantees.
- CONFERENCE PUBLICATION. Collected papers from scientific and technical conferences, symposia, seminars, or other meetings sponsored or cosponsored by NASA.

- SPECIAL PUBLICATION. Scientific, technical, or historical information from NASA programs, projects, and missions, often concerned with subjects having substantial public interest.

- TECHNICAL TRANSLATION. Englishlanguage translations of foreign scientific and technical material pertinent to NASA's mission.

Specialized services that complement the STI Program Office's diverse offerings include creating custom thesauri, building customized databases, organizing and publishing research results ... even providing videos.

For more information about the NASA STI Program Office, see the following:

- Access the NASA STI Program Home Page at http://www.sti.nasa.gov

- E-mail your question via the Internet to help@sti.nasa.gov

- Fax your question to the NASA Access Help Desk at 301-621-0134

- Telephone the NASA Access Help Desk at 301-621-0390

- Write to:

NASA Access Help Desk

NASA Center for AeroSpace Information 7121 Standard Drive

Hanover, MD 21076 
NASA/TM-2003-212314

\section{A 3-D CE/SE Navier-Stokes Solver With Unstructured Hexahedral Grid for Computation of Near Field Jet Screech Noise}

Ching Y. Loh and Ananda Himansu

Taitech, Inc., Beaver Creek, Ohio

Lennart S. Hultgren

Glenn Research Center, Cleveland, Ohio

Prepared for the

Nineth Aeroacoustics Conference and Exhibit cosponsored by the American Institute of Aeronautics and Astronautics and the Confederation of European Aerospace Societies

Hilton Head, South Carolina, May 12-14, 2003

National Aeronautics and

Space Administration

Glenn Research Center 


\section{Acknowledgments}

This work received support from the Supersonic Propulsion Technology Project Office at NASA Glenn Research Center.

This report contains preliminary

findings, subject to revision as analysis proceeds.

The Propulsion and Power Program at

NASA Glenn Research Center sponsored this work.

Available from

NASA Center for Aerospace Information 7121 Standard Drive

Hanover, MD 21076
National Technical Information Service 5285 Port Royal Road

Springfield, VA 22100

Available electronically at http:/ /gltrs.grc.nasa.gov 


\section{A 3-D CE/SE NAVIER-STOKES SOLVER WITH UNSTRUCTURED HEXAHEDRAL GRID FOR COMPUTATION OF NEAR FIELD JET SCREECH NOISE}

\author{
Ching Y. Loh* and Ananda Himansu \\ Taitech, Inc. \\ Beaver Creek, Ohio 45430
}

\author{
Lennart S. Hultgren ${ }^{\dagger}$ \\ National Aeronautics and Space Administration \\ Glenn Research Center \\ Cleveland, Ohio 44135
}

\begin{abstract}
A 3-D space-time CE/SE Navier-Stokes solver using an unstructured hexahedral grid is described and applied to a circular jet screech noise computation. The present numerical results for an underexpanded jet, corresponding to a fully expanded Mach number of 1.42 , capture the dominant and nonaxisymmetric ' $\mathrm{B}$ ' screech mode and are generally in good agreement with existing experiments.
\end{abstract}

\section{Introduction}

Jet noise is one of the most important topics in computational aeroacoustics. Many of its aspects are of primary practical importance and the associated complicated physical phenomena are the topic of many experimental and theoretical investigations. Refs. [1-4] provide a comprehensive discussion and further references. An over/under-expanded supersonic jet emits mixing noise, broadband shock-associated noise, as well as screech tones under certain conditions. The mixing noise is directly associated with large-scale structures, or instability waves, in the jet shear layer; whereas, the broadband shock-associated noise and screech tones are associated with the interaction of these waves with the shock-cell structure in the jet core. The screech tones arise due to a feedback loop, i.e., some of the acoustic waves generated by the wave/shock-cell interaction propagate upstream and regenerate the instability waves at, or in the vicinity of, the nozzle lip. The feedback loop leading to screech tones is sensitive to small changes in the system conditions, and the understanding of the phenomena is to date based mostly on experimental observations [5-8].

\footnotetext{
* Member AIAA

$\dagger$ Associate Fellow AIAA
}

Although substantial progress in numerical computation of jet mixing noise has been made, reliable direct numerical simulation of jet screech noise has up to quite recently not been feasible. Shen and Tam $[9,10]$ obtained excellent results in direct numerical simulations of screech for circular jets using the well-known DRP scheme. In their 3-D computation [10], a spectral method was adopted in the azimuthal direction, and by using only a limited number of spectral functions substantial savings of computer memory and CPU time was achieved, without deterioration of accuracy. Other recent computational work includes [11].

The present authors [12-14] successfully computed axisymmetric near-field screech noise for round jets using the recent $\mathrm{CE} / \mathrm{SE}$ (space-time conservation-element and solution-element) method utilizing (unstructured) triangulated grids. Because of the implementation (based on flux balance) of the non-reflecting boundary conditions (NRBC), a much smaller near field computational domain can be used with this method. However, when the CE/SE method is applied to a 3-D rectangular jet screech noise computation [15], a major challenge emerges. Namely, the number of unstructured tetrahedral cells required to achieve a certain resolution could be as high as tens of millions, which is currently somewhat beyond the capability of common parallel computers such as Linux PC clusters. An alternative is to employ an unstructured hexahedral grid. The number of cells may then be greatly reduced without much loss of resolution. The 3-D CE/SE Navier-Stokes (N-S) solver needs to be modified to accommodate the unstructured hexahedral grid, however.

A general description of the CE/SE Euler method with a hexahedral grid was first given by Zhang et al [16]. In the present paper, the current 3-D CE/SE N-S solver using an unstructured hexahedral grid is described in $\S 2$. The parallel computation implementation of the scheme is outlined in $\S 3$. A 3-D circular jet screech problem is described in $\S 4$. The numerical results are presented and compared with the available experimental data in $\S 5$. Concluding remarks are presented in $\S 6$. 


\section{The Numerical Scheme}

As stated in $\S 1$, for fully 3-D computations of complicated problems such as the jet noise problem, a large number of computational cells are needed to provide sufficient numerical resolution. This imposes severe memory and speed requirements on the computer system. Of course, the choice of unstructured grid type to be used in the computation greatly affects these demands. It turns out that the use of an unstructured hexahedral grid in the $\mathrm{CE} / \mathrm{SE}$ scheme rather than the heretofore standard tetrahedral one leads to significant reductions of the memory and CPU time requirements. Its drawback, of course, is a modest increase in dissipation in the scheme. Zhang et al [16] first presented a 3-D CE/SE Euler method using hexahedral cells instead of tetrahedral ones and provided a detailed description of the implementation.

In general, the CE/SE method [17, 18] systematically solves a set of integral equations derived directly from the physical conservation laws and naturally captures shocks and other discontinuities in the flow. In order to have a compact cell stencil, both conservative variables and their derivatives are computed simultaneously as unknowns. A brief sketch of the 3-D CE/SE scheme with hexahedral cells is given below.

\subsection{Conservation Form of the 3-D Unsteady Compressible Navier-Stokes Equations}

Consider a dimensionless conservation form of the unsteady 3-D Navier-Stokes equations of a perfect gas. Let $\rho, u, v, w, p$, and $\gamma$ be the density, streamwise transverse and spanwise velocities, static pressure, and constant specific heat ratio, respectively. The 3-D NavierStokes equations then can be written in the following vector form:

$$
\boldsymbol{U}_{t}+\boldsymbol{F}_{x}+\boldsymbol{G}_{y}+\boldsymbol{H}_{z}=\mathbf{0},
$$

where $x, y$, and $z$ are the streamwise, transverse, and spanwise coordinates, and $t$ is time. The five components of the conservative flow variable vector $\boldsymbol{U}$ are given by

$$
\begin{gathered}
U_{1}=\rho, \quad U_{2}=\rho u, \quad U_{3}=\rho v, \quad U_{4}=\rho w, \\
U_{5}=p /(\gamma-1)+\rho\left(u^{2}+v^{2}+w^{2}\right) / 2 .
\end{gathered}
$$

The flux vectors in the $x, y$, and $z$ directions, $\boldsymbol{F}, \boldsymbol{G}$, and $\boldsymbol{H}$, respectively, are further split into inviscid and viscous fluxes,

$$
\boldsymbol{F}=\boldsymbol{F}_{i}-\boldsymbol{F}_{v}, \quad \boldsymbol{G}=\boldsymbol{G}_{i}-\boldsymbol{G}_{v}, \quad \boldsymbol{H}=\boldsymbol{H}_{i}-\boldsymbol{H}_{v},
$$

where the inviscid fluxes are

$$
\begin{gathered}
F_{i 1}=U_{2}, \\
F_{i 2}=(\gamma-1) U_{5}+\left[(3-\gamma) U_{2}^{2}-(\gamma-1)\left(U_{3}^{2}+U_{4}^{2}\right)\right] / 2 U_{1}, \\
F_{i 3}=U_{2} U_{3} / U_{1}, \quad F_{i 4}=U_{2} U_{4} / U_{1},
\end{gathered}
$$

$$
\begin{gathered}
F_{i 5}=\gamma U_{2} U_{5} / U_{1}-(\gamma-1) U_{2}\left[U_{2}^{2}+U_{3}^{2}+U_{4}^{2}\right] / 2 U_{1}^{2} ; \\
G_{i 1}=U_{3}, \quad G_{i 2}=U_{2} U_{3} / U_{1}, \\
G_{i 3}=(\gamma-1) U_{5}+\left[(3-\gamma) U_{3}^{2}-(\gamma-1)\left(U_{2}^{2}+U_{4}^{2}\right)\right] / 2 U_{1}, \\
G_{i 4}=U_{3} U_{4} / U_{1}, \\
\left.G_{i 5}=\gamma U_{3} U_{5} / U_{1}-(\gamma-1) U_{3}\left[U_{2}^{2}+U_{3}^{2}+U_{4}^{2}\right)\right] / 2 U_{1}^{2} ; \\
H_{i 1}=U_{4}, \quad H_{i 2}=U_{2} U_{4} / U_{1}, \quad H_{i 3}=U_{3} U_{4} / U_{1}, \\
H_{i 4}=(\gamma-1) U_{5}+\left[(3-\gamma) U_{4}^{2}-(\gamma-1)\left(U_{2}^{2}+U_{3}^{2}\right)\right] / 2 U_{1}, \\
\left.H_{i 5}=\gamma U_{4} U_{5} / U_{1}-(\gamma-1) U_{4}\left[U_{2}^{2}+U_{3}^{2}+U_{4}^{2}\right)\right] / 2 U_{1}^{2} ;
\end{gathered}
$$

and the viscous fluxes are

$$
\begin{gathered}
F_{v 1}=0, \quad F_{v 2}=\mu\left(2 u_{x}-\frac{2}{3} \Delta\right), \\
F_{v 3}=\mu\left(u_{y}+v_{x}\right), \quad F_{v 4}=\mu\left(u_{z}+w_{x}\right), \\
F_{v 5}=\mu\left[2 u u_{x}+v\left(u_{y}+v_{x}\right)+w\left(w_{x}+u_{z}\right)-\frac{2}{3} u \Delta+\right. \\
\left.\frac{\gamma}{\operatorname{Pr}} \frac{\partial}{\partial y}\left(\frac{U_{4}}{U_{1}}-\frac{u^{2}+v^{2}}{2}\right)\right], \\
G_{v 1}=0, \quad G_{v 2}=\mu\left(v_{x}+u_{y}\right), \\
G_{v 3}=\mu\left(2 v_{y}-\frac{2}{3} \Delta\right), \\
G_{v 4}=\mu\left(v_{z}+w_{y}\right), \\
G_{v 5}=\mu\left[2 v v_{y}+u\left(u_{y}+v_{x}\right)+w\left(w_{y}+v_{x}\right) w-\frac{2}{3} v \Delta+\right. \\
\left.\frac{\gamma}{\operatorname{Pr}} \frac{\partial}{\partial y}\left(\frac{U_{4}}{U_{1}}-\frac{u^{2}+v^{2}}{2}\right)\right], \\
H_{v 1}=0, \quad H_{v 2}=\mu\left(w_{x}+u_{z}\right), \\
H_{v 3}=\mu\left(v_{z}+w_{y}\right), \quad H_{v 4}=\mu\left(2 w_{z}-\frac{2}{3} \Delta\right), \\
H_{v 5}=\mu\left[2 w w_{z}+u\left(u_{z}+w_{x}\right)+v\left(w_{y}+v_{x}\right)-\frac{2}{3} v \Delta+\right. \\
\left.\frac{\gamma}{\operatorname{Pr}} \frac{\partial}{\partial y}\left(\frac{U_{4}}{U_{1}}-\frac{u^{2}+v^{2}}{2}\right)\right],
\end{gathered}
$$

where $u, v, w, u_{x}, u_{y}, u_{z}$, etc. are the flow velocities and their spatial derivatives, which can be expressed in terms of the conservative variables $U_{1}, U_{2}$, etc. along with their gradients. $\operatorname{Pr}=0.72$ is the Prandtl number, $\mu$ the kinematic viscosity, and

$$
\Delta=u_{x}+v_{y}+w_{z}
$$

is the velocity divergence.

By considering $(x, y, z, t)$ as coordinates of a fourdimensional Euclidean space, $E_{4}$, and using Gauss' divergence theorem, it follows that Eq. (1) is equivalent to the following integral conservation law:

$$
\oint_{S(V)} \boldsymbol{I}_{m} \cdot \mathrm{d} \boldsymbol{S}=\mathbf{0}, \quad m=1,2,3,4,5,
$$

where $S(V)$ denotes the surface around a volume $V$ in $E_{4}$ and $\boldsymbol{I}_{m}=\left(F_{m}, G_{m}, H_{m}, U_{m}\right)$. 


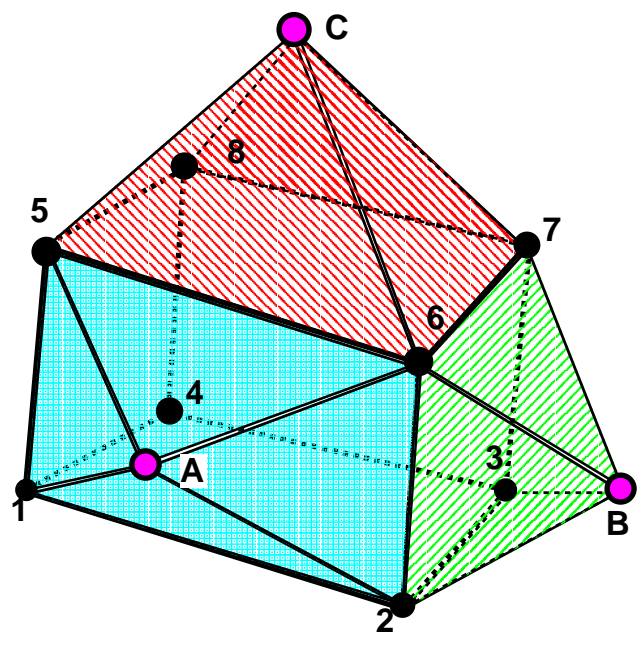

Figure 1: A hexahedral cell with its six neighboring average cell centers (ACs). For clarity, only three of them, $A, B, C$ are shown. Together, they form a polyhedron of 24 faces or the base of the $C V$.

\subsection{Unstructured Hexahedral Grid}

Fig. 1 illustrates a hexahedron cell $1234-5678$ in the unstructured grid. Each of the six quadrilateral surfaces (e.g. 1265, 2376, etc.) is associated with a neighboring hexahedral cell. Note that the four nodes of such a quadrilateral may not lie on the same plane. Assume $A$, $B, C, \ldots$, are the average centers $(A C s)$ of the respective neighboring cells. The coordinates of the average center of a hexahedron are the simple average of the coordinates of its eight vertices. The average center may not necessarily coincide with the geometrical (gravity) center. The eight vertices of each hexahedron cell and the six average centers ( $A, B, C \ldots$, only three are shown in Fig. 1) of the neighboring cells form a polyhedron of 24 faces, which, when incorporating the time $t$ direction, is the finite volume $V$ in $E_{4}$ in Eq. (2) or the control volume $C V$.

\subsection{Compact Updating}

For any explicit time-marching scheme, flow data at the neighboring nodes at the previous time step are required to update the flow data at the current node to the present time level. In a finite difference method, a difference scheme is utilized to do the updating. In most finite volume schemes, the computational cell (e.g., 1234-5678 in Fig. 1) is the control volume $(C V)$. First, the fluxes on the (hyper) surfaces $S(V)$ (e.g., 1265 in Fig. 1) need to be updated. Conventionally, flow data is extrapolated from several neighboring nodes to the center of the hyper-surface. In the CE/SE algorithm, a compact updating is employed [17, 18]. The compact updating achieves high resolution by using a cell stencil consisting only the immediate neighboring cells. For example, the $C V$ is expanded from the hyper-hexahedral cell
$1234-5678$ to a hyper-polyhedron of 24 faces (Fig. 1), with all the six neighboring cell centers $(A, B, C \ldots)$ included as vertices. When applying Eq. (2) to this hyperpolyhedron in $E_{4}$, the flux associated with the face 1265 is now replaced by the fluxes associated with the four triangles $\triangle A 12, \triangle A 26, \triangle A 65$ and $\triangle A 51$ for the new $C V$. The advantage of this procedure is that flow data at the cell center $A$ is known and since $A$ lies in each of these triangles, no extrapolation through the interior of the $C V$ is needed when evaluating the fluxes associated with these triangles. The updating is thus conservative. In the $\mathrm{CE} / \mathrm{SE}$ scheme, in order to achieve higher order accuracy, flow data are extrapolated by Taylor expansion from $A$ to the triangle centers along the triangle surface plane. Consequently, not only the conservative flow variables $\boldsymbol{U}$ but their spatial derivatives also are considered as the unknowns (totally 20 scalar unknowns) Note that $\boldsymbol{U}_{t}$ is obtained by evaluating Eq. (1). The hyper24-face-polyhedron is the control volume where Eq. (2) is applied for conservative updating. Any of these 24 surfaces (segments of solution elements or $S E s$ ) is associated with one of the six neighboring average centers $(A C s$,e.g. $A, B, C, \ldots)$, where the solution $\boldsymbol{U}$ and its gradients are already given at the previous time step $n$. Then both the inviscid and viscous fluxes on these 24 faces (hyperplane surfaces in $E_{4}$ space) can be calculated by evaluating the flux functions $\boldsymbol{F}, \boldsymbol{G}$ and $\boldsymbol{H}$ at the face geometrical centers through Taylor expansion. Consequently, the unknown $\boldsymbol{U}^{n+1}$ at the new time level $n+1$ is evaluated at the geometrical center of the polyhedron from the divergence theorem (2) in $E_{4}$ space. Note that the 'geometrical center' or centroid of the polyhedron is in general different from its average center $(A C)$. After evaluating the new gradients (see next subsection) $U_{x}$, $\boldsymbol{U}_{y}, \boldsymbol{U}_{z}$, it takes only one more step of Taylor expansion to extrapolate $\boldsymbol{U}^{n+1}$ to the average center $(A C)$.

The present compact updating avoids the uncertainty of dimensional-splitting and extrapolation, and hence yields better accuracy. In addition, no Riemann solver is needed at these hyper-surfaces. Incorporation of the gradients of $\boldsymbol{U}$ further enhances the accuracy of the scheme and makes it possible to use a compact cell stencil.

After all the $\boldsymbol{U}^{n+1}$ at the polyhedron centroids are updated, the next step is to calculate their corresponding spatial gradients.

\subsection{Evaluation of Spatial Gradients}

As in the standard CE/SE procedure [17], unknown spatial derivatives/gradients $\boldsymbol{U}_{x}, \boldsymbol{U}_{y}$, and $\boldsymbol{U}_{z}$ are evaluated using the weighted average technique or an extended van Albada limiter [19]. The six neighboring polyhedral centers $A, B, C, D, E, F$ around a hexahedron cell (Fig. 2) form an octahedron. The vertices of each of the triangular face of the octahedron and the polyhedral center $O$ 


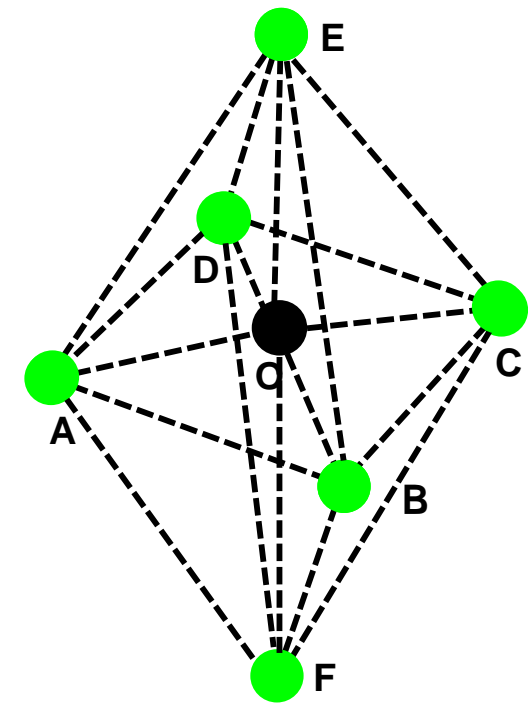

Figure 2: An octahedron formed by the six neighboring polyhedron centroids $A, B, C, D, E, F$, with the current polyhedron cell centroid $O$ in the middle. With $O$ always as a vertex, eight tetrahedra are formed and hence eight sets of gradients exist.

associated with the current hexahedral cell form a tetrahedron (e.g. $A B E O$ in Fig. 2). The $\boldsymbol{U}^{n+1}$ are already calculated at all the vertices. For each tetrahedron, a set of $\boldsymbol{U}_{x}^{n+1}, \boldsymbol{U}_{y}^{n+1}$, and $\boldsymbol{U}_{z}^{n+1}$ can be directly calculated by solving a linear equation system. Totally, there are eight different tetrahedra, and so also eight sets of the spatial derivative data. The final values of the spatial derivatives are obtained by applying the extended van Albada limiter (weighted average) to these eight sets of data. Let $\boldsymbol{U}_{x}{ }^{(i)}$, $\boldsymbol{U}_{y}{ }^{(i)}, \boldsymbol{U}_{z}{ }^{(i)}, i=1,2, \ldots, 8$ be respectively the gradients from the $i$ th set data. Let

$$
\tau_{i}=\left[\left(\boldsymbol{U}_{x}{ }^{(i)}\right)^{2}+\left(\boldsymbol{U}_{y}{ }^{(i)}\right)^{2}+\left(\boldsymbol{U}_{z}{ }^{(i)}\right)^{2}\right]^{\alpha},
$$

where $\alpha$ is any real index number. Let $t_{i}=\tau_{1}{ }^{-1}$; if $\tau_{i}=0, t_{i}=0$ and that

$$
t=t_{1}+t_{2}+t_{3}+\ldots+t_{8},
$$

then, with the van Albada limiter,

$$
\boldsymbol{U}_{x}=\frac{\Sigma \boldsymbol{U}_{x}{ }^{(i)} t_{i}}{t}, \boldsymbol{U}_{y}=\frac{\Sigma \boldsymbol{U}_{y}{ }^{(i)} t_{i}}{t}, \boldsymbol{U}_{z}=\frac{\Sigma \boldsymbol{U}_{z}{ }^{(i)} t_{i}}{t} .
$$

As is well-known, the van Albada limiter is less diffusive. In practice, it was found that if the power index number $\alpha$ is chosen to be slightly negative, say, $\alpha=-0.2$, the numerical dissipation resulting from averaging could be further reduced.

Once the new gradients are evaluated, $\boldsymbol{U}^{n+1}$ at the $A C$ of the hexahedron is obtained by Taylor expansion from the centroid of the polyhedron. One marching step is thus completed.
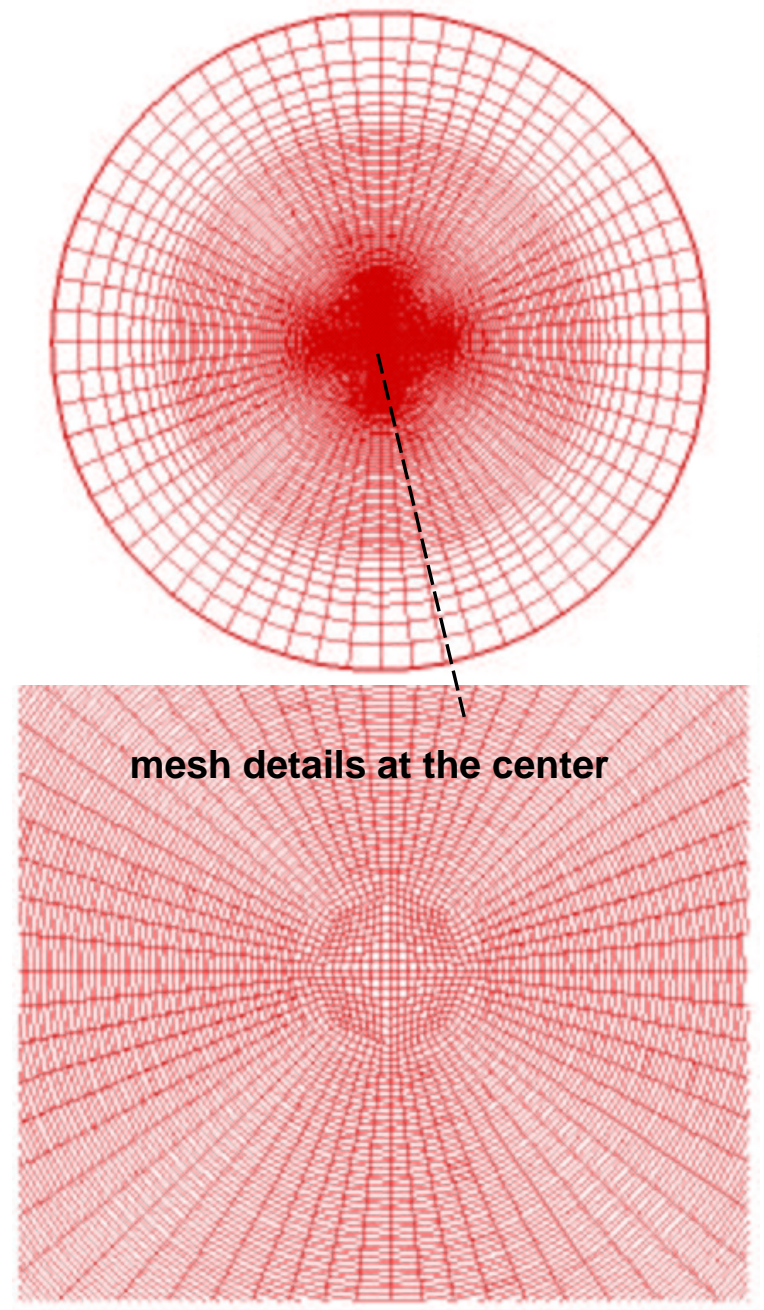

Figure 3: 2-D quadrilateral mesh in a cross section in a 3-D hexahedral grid

\section{Hexahedral Grid Generation and Parallel Computation}

In order to substantially reduce the required number of cells and to increase numerical stability in the computation, a hexahedral grid is adopted for the 3-D CE/SE Navier-Stokes (N-S) solver. For the simple geometry of a circular jet, with the $x$ axis assumed in the stream direction, the hexahedral grid can be generated as follows:

1. a 2-D quadrilateral unstructured mesh is generated in a circular domain on the $y-z$ plane (Fig. 3);

2. the 2-D mesh is translated step by step in the $x$ direction to form the 3-D hexahedral mesh. At locations that are occupied by the jet nozzle body, no cells are generated.

In the present 3-D screech noise computation, employing a hexahedral grid instead of a tetrahedral one helps to 


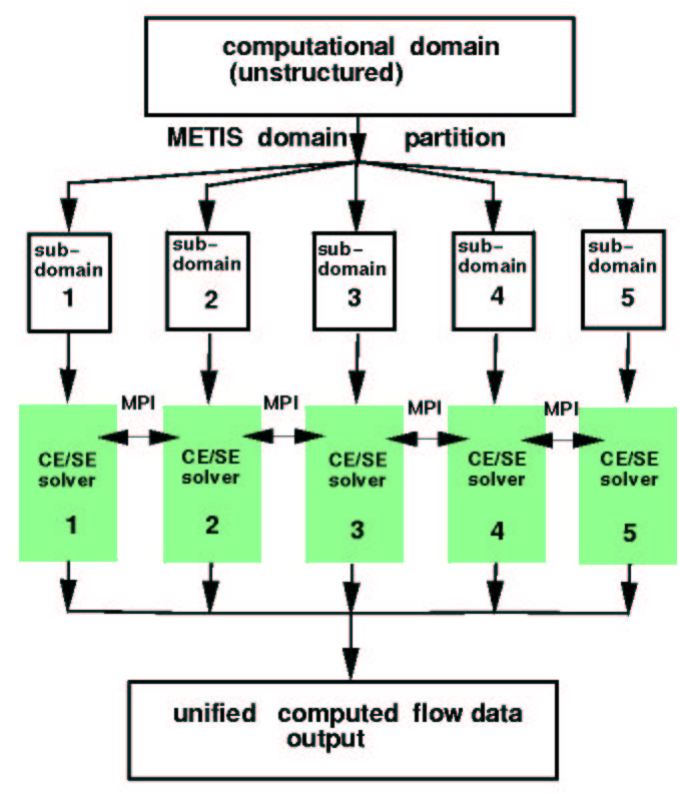

Figure 4: Schematic diagram of parallel computation.

reduce the number of cells from tens of millions to a few millions. Still, the number of computational cells is very large. Due to the large number of computational cells (about 3.67 millions in this case), parallel computation with multiple processors becomes necessary in view of computation turn-around time and memory size.

The parallelization procedure is similar to the one described in [15] and is sketched in Fig. 4:

1. the unstructured hexahedral grid is generated for the entire computational domain;

2. the domain is decomposed into subdomains according to the assigned number of processes (usually one-to-one with CPU's, here 60 are used), using the METIS code. METIS is an efficient mesh partitioning code and is freely available from the University of Minnesota [20] - for example, Fig. 5 illustrates a typical partition of the computational domain for the current circular jet noise problem;

3. the N-S flow solver is modified to use MPI library calls and applied to each subdomain to conduct computations, with neighboring domains exchanging pertinent results.

MPI, or message passing interface, is an interprocessor communication protocol standard. The software library is prepared by the Argonne National Laboratory [21].

\section{The 3-D Jet Screech Noise Problem}

The circular jet in 3-D space is sketched in Fig. 6. The flow at the nozzle exit is choked, i.e., the nozzle exit

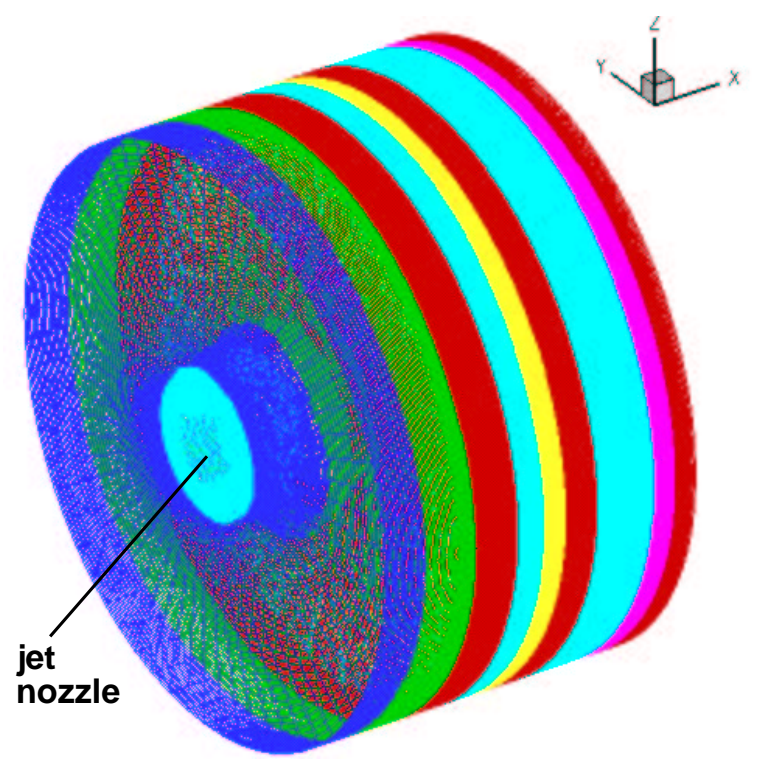

Figure 5: A typical partition of the computational domain by METIS, with different shading or color indicating the subdomains.

Mach number, $M_{e}$, is unity, and the ambient air is stationary. The case of jet Mach number $M_{j}=1.42$ is considered. These conditions correspond to the experimental conditions of Panda [5, 6]. In these experiments, it was shown that for $M_{j}=1.42$, the jet noise field exhibits truly 3-D phenomena, e.g., the flapping 'B' mode. Hence, a 3-D Navier-Stokes solver described above is required.

In the investigation, our attention is focussed on the near field of the nozzle since this is the source region of the noise. The inner diameter, $D$, of the jet nozzle is chosen as the length scale. The density, $\rho_{0}$, speed of sound, $a_{0}$, and temperature $T_{0}$ in the ambient flow are taken as scales for the dependent flow variables.

In order to clearly display the upstream propagating screech waves, the computational domain was extended $2 D$ upstream of the nozzle exit. The full computational domain is a circular cylinder of $10 D$ axial length and $7.5 \mathrm{D}$ radius. At the nozzle exit, the inflow plane is recessed by two cells so as not to numerically restrict or influence the feed-back loop. A straight nozzle lip of $0.25 \mathrm{D}$ in thickness is adopted in the computations. Note that in the experimental setup of Panda [5, 6], the actual nozzle diameter $D=25.4 \mathrm{~mm}$ and the nozzle exit lip is beveled.

The unstructured hexahedral grid currently used is generated as described in $\S 2$. The hexahedral cells are non-uniform since good grid resolution is needed in the jet shear layer and in the screech feedback loop paths. Cell numbers in the $x$ and radial directions are typically 


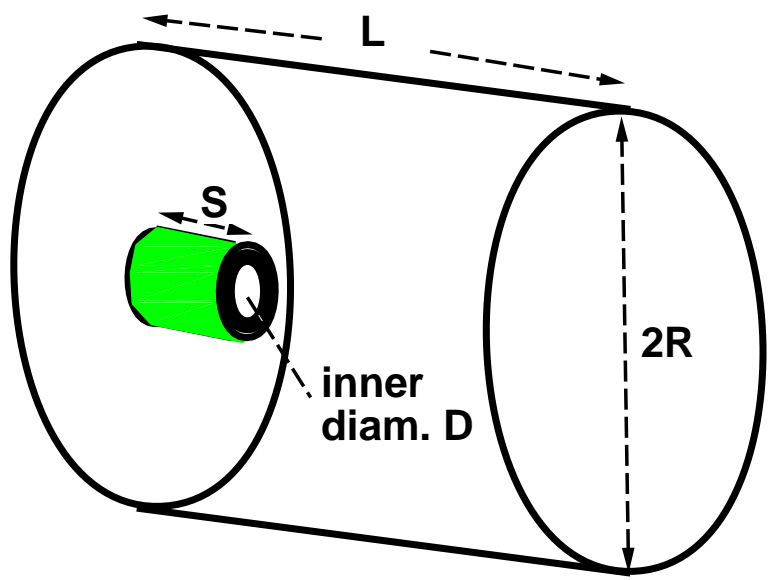

Figure 6: Geometry of the computational domain: the inner diameter of the nozzle is $D$; the nozzle extends into the computational domain by $S=2 D$, the domain length $L=8 D$ and the radius is $R=5.5 D$ (sponge zones are excluded)

400 and 160 respectively. In the azimuthal direction, there are 64 cells, corresponding to totally about 3.67 million hexahedral cells. The last 6 cells in the streamwise and radial directions have exponentially growing size, respectively, and serve as sponge zones to substantially eliminate any small remaining numerical reflection from the outflow and circumferential non-reflecting boundaries.

\subsection{Initial Conditions}

Initially, the flow of the entire domain is set at the ambient flow conditions (using nondimensional variables), i.e.,

$$
\begin{gathered}
\rho_{0}=1, \quad p_{0}=\frac{1}{\gamma}, \\
u_{0}=0, \quad v_{0}=0, \quad w_{0}=0 .
\end{gathered}
$$

\subsection{Boundary Conditions}

At the inlet boundary, the conservative flow variables and their spatial derivatives are specified to be the same as the ambient flow, except at the nozzle exit, where an elevated pressure is imposed, i.e., the jet is under-expanded, as in the physical experiments. By using the ideal gas isentropic relations, it follows that the nondimensional flow variables at the nozzle exit, with $M_{e}=1$, are given by

$$
\begin{gathered}
\rho_{e}=\frac{\gamma(\gamma+1) p_{e}}{2 T_{r}}, \\
p_{e}=\frac{1}{\gamma}\left[\frac{2+(\gamma-1) M_{j}^{2}}{\gamma+1}\right]^{\frac{\gamma}{\gamma-1}}, \\
u_{e}=\left(\frac{2 T_{r}}{\gamma+1}\right)^{1 / 2}, \quad v_{e}=0, \quad w_{e}=0,
\end{gathered}
$$

where $T_{r}$ is the reservoir (plenum) temperature. We will also follow the experimental cold-flow condition where the reservoir temperature equals the ambient one, i.e., $T_{r}=1$.

At the circumferential and outflow boundaries, the Type II and Type I CE/SE non-reflecting boundary conditions as described in the next subsection are imposed, respectively. The no-slip boundary condition is applied on all the nozzle walls.

\subsection{Non-Reflecting Boundary Conditions}

In the $\mathrm{CE} / \mathrm{SE}$ scheme, non-reflecting boundary conditions (NRBC) can be easily constructed based on planewave propagation theory for hyperbolic conservation laws [22]. There are various implementations of the non-reflecting boundary condition (NRBC) and in general they have proven to be well suited for aeroacoustic problems [14, 23]. The following 3-D NRBCs are employed in this paper.

For a ghost grid node $(j, n)$ lying at the outer radius of the domain the non-reflective boundary condition (Type II) requires that

$$
\left(\boldsymbol{U}_{x}\right)_{j}^{n}=\left(\boldsymbol{U}_{y}\right)_{j}^{n}=\left(\boldsymbol{U}_{z}\right)_{j}^{n}=0,
$$

while $\boldsymbol{U}_{j}^{n}$ is kept fixed at the initial steady boundary value. At the downstream boundary, where there are substantial gradients in the radial direction, the nonreflective boundary condition (Type I) requires that

$$
\left(\boldsymbol{U}_{x}\right)_{j}^{n}=0
$$

while $\boldsymbol{U}_{j}^{n},\left(\boldsymbol{U}_{y}\right)_{j}^{n}$ and $\left(\boldsymbol{U}_{z}\right)_{j}^{n}$ are now defined by simple extrapolation from the nearest interior node $j^{\prime}$, i.e.,

$$
\begin{gathered}
\boldsymbol{U}_{j}^{n}=\boldsymbol{U}_{j^{\prime}}^{n}, \\
\left(\boldsymbol{U}_{y}\right)_{j}^{n}=\left(\boldsymbol{U}_{y}\right)_{j^{\prime}}^{n}, \quad\left(\boldsymbol{U}_{z}\right)_{j}^{n}=\left(\boldsymbol{U}_{z}\right)_{j^{\prime}}^{n} .
\end{gathered}
$$

As will be observed later, these NRBCs, when combined with the above buffer/sponge zones, are robust enough to allow a near field computation without disturbing or distorting the flow and acoustic fields.

\section{Numerical Results}

In this section, 3-D numerical results for the underexpanded circular jet described above are presented and compared to experimental results $[5,6]$. Computations are conducted for the jet Mach number $M_{j}=1.42$. At this moderate jet Mach number, the dominant unsteady motion in the experiments, see [5], is truly three dimensional. With an appropriate time step size, a large number $(510,000)$ of time steps are performed in order to achieve sufficient accuracy in the Fourier analysis of time series data. Note that no harmonic forcing is imposed in the numerical simulation. The initial impact of the boundary condition at the nozzle exit stimulates the jet shear layer and triggers the feedback loop that generates the (then) self-sustained screech waves. 

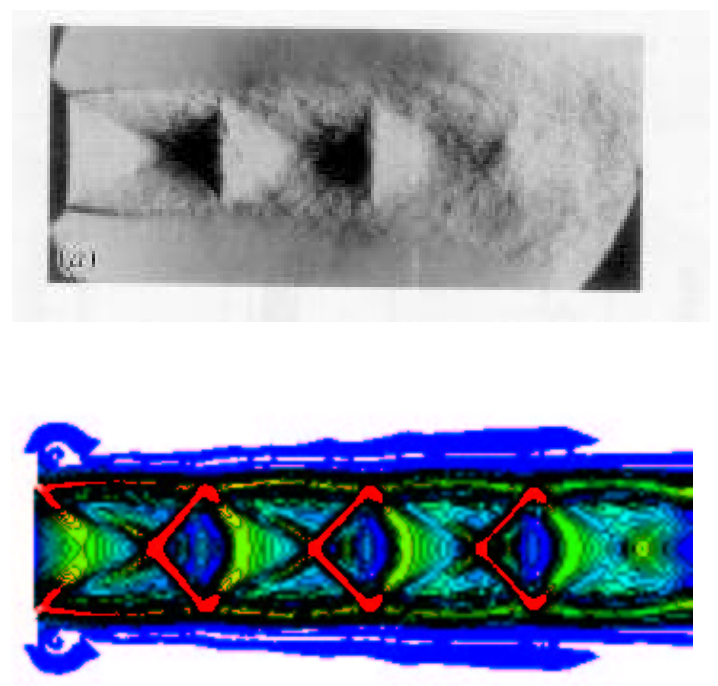

Figure 7: Time-averaged experimental (top) and instantaneous numerical (bottom) Schlieren pictures, showing shock cell structure; $M_{j}=1.42$.

\subsection{Shock-Cell Structure}

Experimental results for jets are often documented in terms of Schlieren pictures. It is straightforward to construct Schlieren plots (density-gradient modulus) from the numerical results. For the case of $M_{j}=1.42$, Fig. 7 shows the experimental [5, Fig. 4(a)] and numerical Schlieren pictures. Good agreement in shockcell structure is shown. For example, the shock cell width (spacing) is about $1.28 \mathrm{D}$ in the streamwise direction. Note that the experimental Schlieren plot is a timeaveraged results, while the numerical one is a snapshot at a relatively early stage (time step 110,000). In the experimental Schlieren plot, it is observed that the first shock cell appears to be sharp and clear since the shear-layer instability wave is too weak at this location to significantly affect the shock cell. However, once the instability wave has gained a sufficient amplitude through its streamwise growth, it interacts strongly with the shock cells. This is evident from the deformations from the second shock cell and onwards and the additional blurring due to timeaveraging in top panel of Fig. 7.

\subsection{Near-Field Radiating Screech Waves}

Figs. 8- 12, which represent a series of instantaneous snapshots of pressure iso-surfaces in the flow field, illustrate the generation and propagation of screech waves. Since no forcing is applied in the numerical simulation, these waves are a clear indication of a self-sustained oscillation. For $M_{j}=1.42$, the jet screech is in the flapping B mode, which is a truly three dimensional one. The screech waves not only propagate in the upstream direction but also swirl and flap around the jet core.

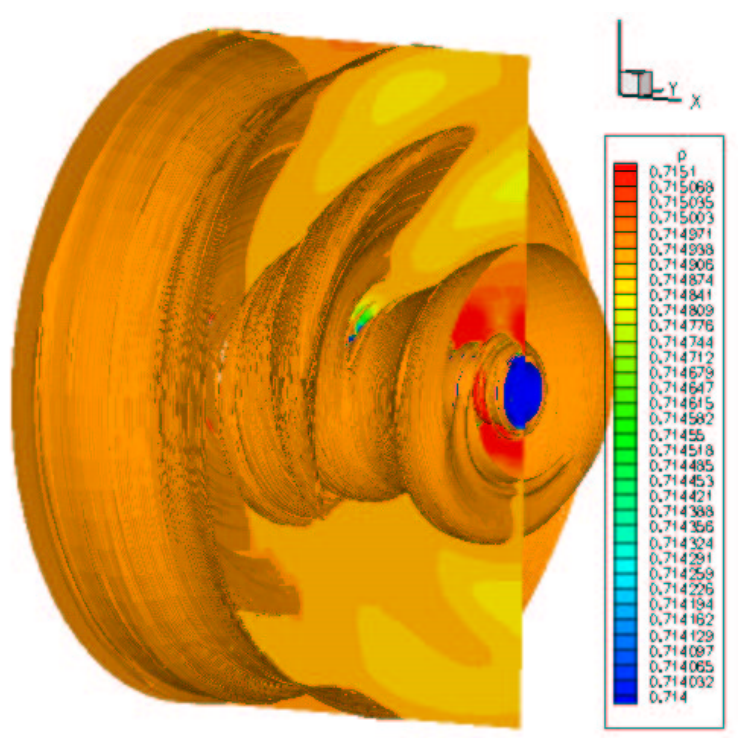

Figure 8: Pressure iso-surfaces at time step 120,000; a $x-z$ cross section slice is also shown.

After the computation has run for about 110,000 time steps, truly three dimensional asymmetric wave patterns begin to appear. Fig. 8 and Fig. 9 demonstrate the pressure iso-surfaces at time steps 120,000 and 130,000 respectively and from different angle. The iso-surfaces clearly take on nonaxisymmetric forms. However, at time steps of 200,000 and 320,000, the pressure isosurfaces again turn out to look more axisymmetric, as demonstrated in Fig. 10 and Fig. 11 respectively. At time step 510,000, when the computation was stopped, a highly asymmetric pattern can be observed, see Fig. 12.

\subsection{Screech Frequency and Sound Pressure Level (SPL)}

The numerical time history is recorded for the location $(2.0,0.6,0)$ at the nozzle lip in the flow field and later post-processed to obtain spectral information using Fast Fourier Transform (FFT) techniques. The recording begins after an initial time period has elapsed (180, 000 actual time steps) ensuring that any start-up transients have left the computational domain. Figure 13 displays the SPL for the case of $M_{j}=1.42$. The SPL plot shows the spikes at the fundamental ' $\mathrm{B}$ ' mode frequency and its harmonics. The fundamental frequency spike correspond to screech frequency of $4500 \mathrm{~Hz}(\mathrm{SPL}=122 \mathrm{~dB})$, which agrees quite well with the experimental value of $4350 \mathrm{~Hz}$.

\subsection{Performance of the Parallel Computation}

The computation was mainly carried out on a Linux Pentium III PC cluster (NASA Glenn Research Center CW-7 cluster) using 60 processes, running on 20 CPUs. Normally, one would want a one-to-one relationship between 


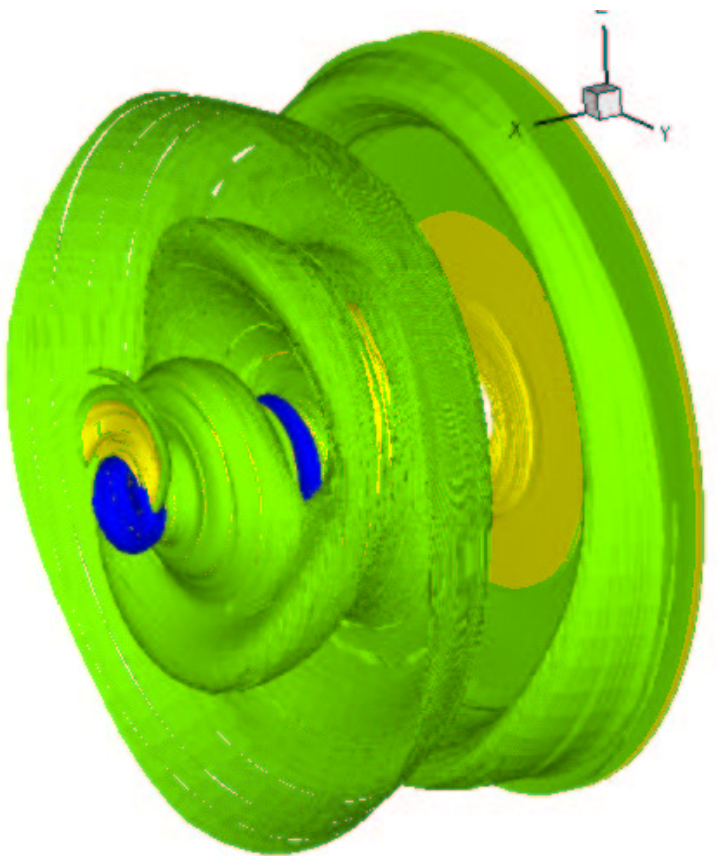

Figure 9: Pressure iso-surfaces at time step 130,000.

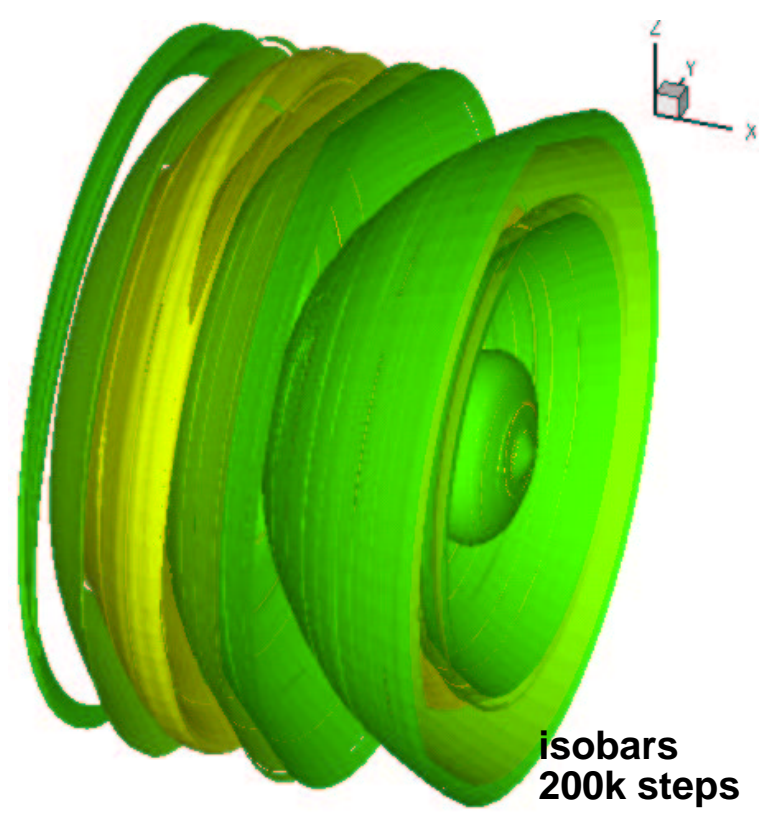

Figure 10: Pressure iso-surfaces at time step 200,000.

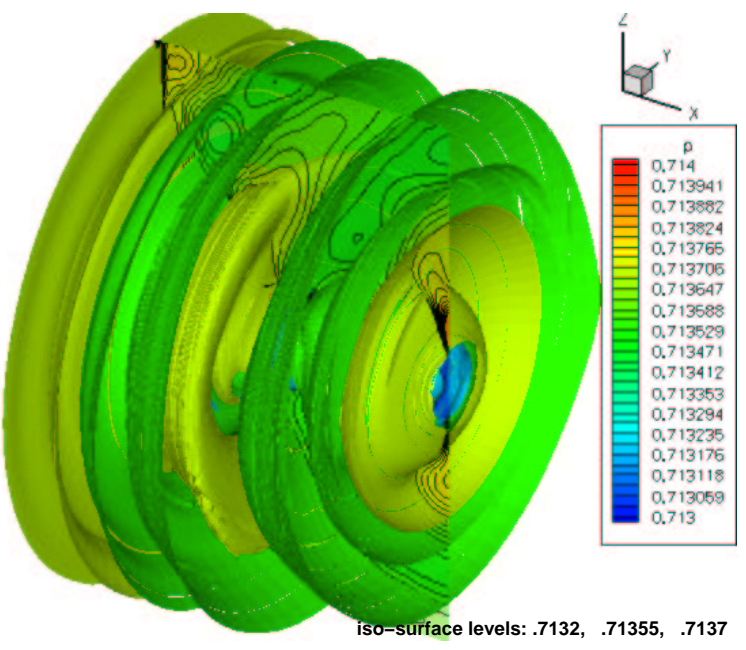

Figure 11: Pressure iso-surfaces at time step 320,000; a $x-z$ cross section slice is also shown.

the number of processes and processors. This less than optimal situation lead to about $15 \%$ increase in the wallclock time and was due to the computation having been started on a different Linux PC cluster using 60 CPUs. In the optimal situation, it takes about 8 seconds wallclock time to march one step on the $\mathrm{CW}-7$ cluster. The parallel computation was also tested on the SGI (Silicon Graphics ) Origin 3800 workstation cluster with 64 and 128 processors at NASA Ames Research Center. From 64 to 128 processors, the clock time reduces linearly with the increasing number of processors, but the reduction will begin to level off if more processors are used. As a result of the explicit time-marching in the scheme, when running with 64 processors, the number of MFLOPS (megaflops) per processor remains between 170 and 183, exceeding $20 \%$ (160) of the theoretical peak MFLOPS. This performance is considered excellent by code-performance specialists at NASA Ames.

\section{Concluding Remarks}

In this paper, a 3-D CE/SE N-S solver using an unstructured hexahedral grid is briefly described and tested in a 3-D circular jet screech problem. The use of a hexahedral grid rather than a tetrahedral one enhances the numerical stability of the scheme and significantly reduces both memory size and CPU time, making the CE/SE scheme a viable tool for near-field CAA simulation.

For the test case of $M_{j}=1.42$, the jet screech frequency of the dominant nonaxisymmetric ' $\mathrm{B}$ ' mode and the shock cell structure agree well with the experimental data $[5,6]$. Perhaps due to the relatively coarse grid used, the computed SPL is somewhat lower than the experimental one. Further tests with refined grids are being carried out and will be reported in the future. 


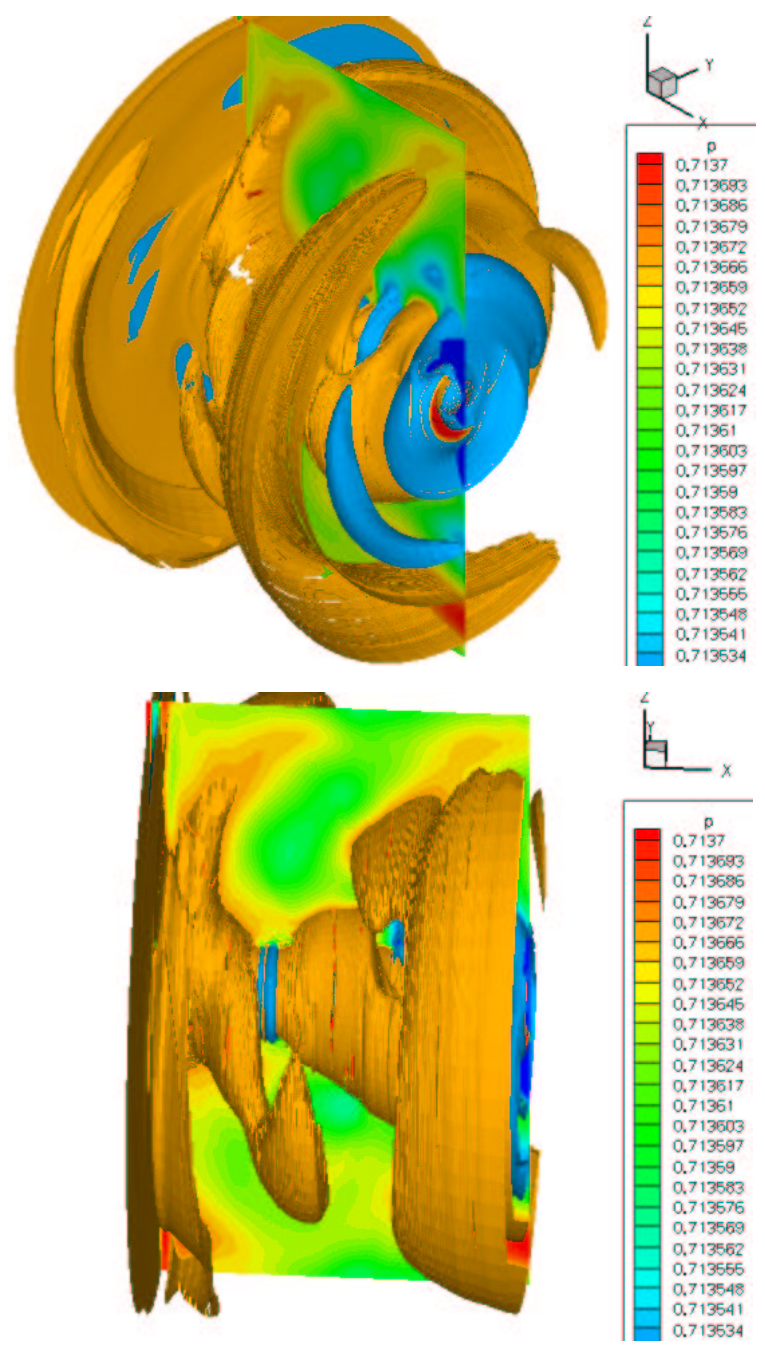

Figure 12: Pressure iso-surfaces at time step 510,000 viewed from two different angles; $x-z$ cross section slices are also shown.

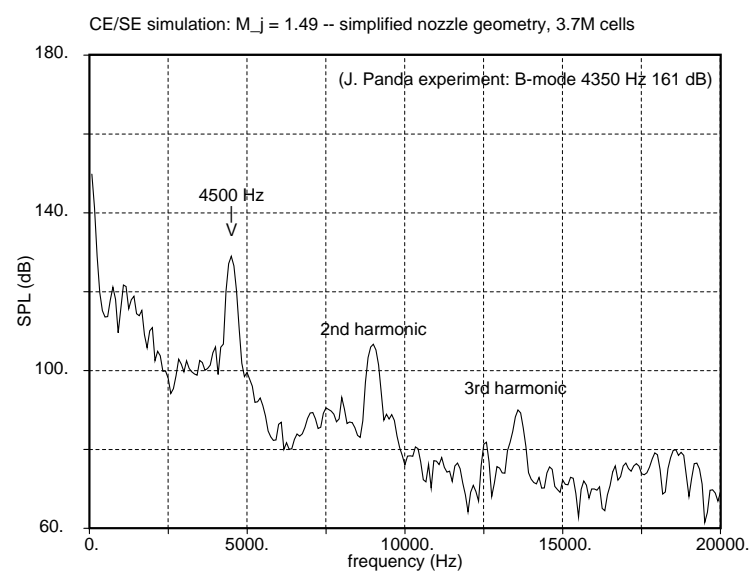

Figure 13: SPL at nozzle wall.

\section{References}

[1] Tam, C. K .W., "Supersonic Jet Noise,” Ann. Rev. Fluid Mech. vol. 27, pp. 17-43 (1995).

[2] Seiner, J. M., "Advances in High Speed Jet Aeroacoustics," AIAA Paper 84-2275 (1984).

[3] Tam, C. K. W., "Jet Noise Generated by Large Scale Coherent Motion," NASA RP-1258, pp. 311390 (1991).

[4] Raman G., "Advances in Understanding Supersonic Jet Screech: Review and Perspective," Prog. Aerosp. Sci. vol. 34, pp. 45-106 (1998).

[5] Panda, J., "Shock Oscillation in Underexpanded Screeching Jets," J. Fluid Mech., vol. 363, pp. 173198 (1998).

[6] Panda, J., "An Experimental Investigation of Screech Noise Generation," J. Fluid Mech., vol. 378, pp. 71-96 (1999).

[7] Panda, J., Raman, G. and Zaman, K. B. M. Q., "Underexpanded Screeching Jets from Circular, Rectangular and Elliptic Nozzles", AIAA paper 971623 (1997).

[8] Ponton, M. K., Seiner, J. M. and Brown, M. C., “ Near Field Pressure Fluctuations in the Exit Plane of a Choked Axisymmetric Nozzle", NASA TM 113137 (1997).

[9] Shen, H. and Tam, C. K. W., "Numerical Simulation of the Generation of Axisymmetric Mode Jet Screech Tones," AIAA Paper 98-0283 (1998).

[10] Shen, H. and Tam, C. K. W., "Three-Dimensional Numerical Simulation of the Jet Screech Phenomenon" AIAA Paper 2001-0820 (2001).

[11] Imamoglu, B. and Balakumar, P. "Computation of Shock Induced Noise in Imperfectly Expanded Supersonic jets", AIAA Paper 2002-2527 (2002).

[12] Loh, C. Y., Hultgren, L. S., and Jorgenson, P. C. E., "Near Field Screech Noise Computation for an Underexpanded jet by the CE/SE Method," AIAA Paper 2001-2252 (2001).

[13] Loh, C. Y. and Hultgren, L. S., "Computing Jet Screech - A Complex Aeroacoustic Feedback System", presented at the 2nd Int'l Conference on CFD (ICCFD2), also NASA/TM-2002-211807 (2002).

[14] Jorgenson, P. C. E. and Loh, C. Y., "Computing Axisymmetric Jet Screech Tones Using Unstructured Grids", AIAA Paper 2002-3889 (2002). 
[15] Loh, C. Y., Himansu, A. Wang, X.-Y. and Jorgenson, P. C. E., "Computation of an Underexpanded 3-D Rectangular jet by the CE/SE Method", AIAA Paper 2001-0986 (2001).

[16] Zhang, Z.-C., Yu, S. T. and Chang, S.-C., "A SpaceTime Conservation Element and Solution Element method for Solving the Two and Three Dimensional Unsteady Euler Equations Using Quadrilateral and Hexahedral Meshes", J. Comp. Phys. vol. 175, pp. 168-199 (2002).

[17] Chang, S.-C., Wang, X.-Y. and Chow, C.-Y., "The Space-Time Conservation Element and Solution Element Method-A New High Resolution and Genuinely Multidimensional Paradigm for Solving Conservation Laws," J. Comp. Phys. vol. 159, pp. 89-136 (1999).

[18] Wang, X.-Y. and Chang S.-C., “ A 2-D Nonsplitting Unstructured Triangular Mesh Euler Solver Based on the Space-Time Conservation Element and Solution Element Method" C.F.D. J. vol. 8, pp. 309-325 (1999).

[19] van Albada, G. D., van Leer, B. and Roberts, W. W., "A Comparative Study of Computational Methods in Cosmic Gas Dynamics," Astronomy and Astrophys., vol. 108, pp. 76-84 (1982).

[20] Karypis, G. and Kumar, V. "Multilevel k-way Partitioning Scheme for Irregular Graphs", Univ. of Minnesota Dept. of Comp. Sc./Army HPC Research Center Tech. Report 95-064 (1995).

[21] website: http://www-unix.mcs.anl.gov/mpi

[22] Loh, C. Y., "On a Nonreflecting Boundary Condition for Hyperbolic Conservation Laws," AIAA Paper 2003-3975 (2003).

[23] Loh, C. Y., Hultgren, L. S. and Chang S.-C., "Computing Waves in Compressible Flow Using the Space-Time Conservation Element Solution Element Method," AIAA J., Vol. 39, pp. 794-801 (2001). 


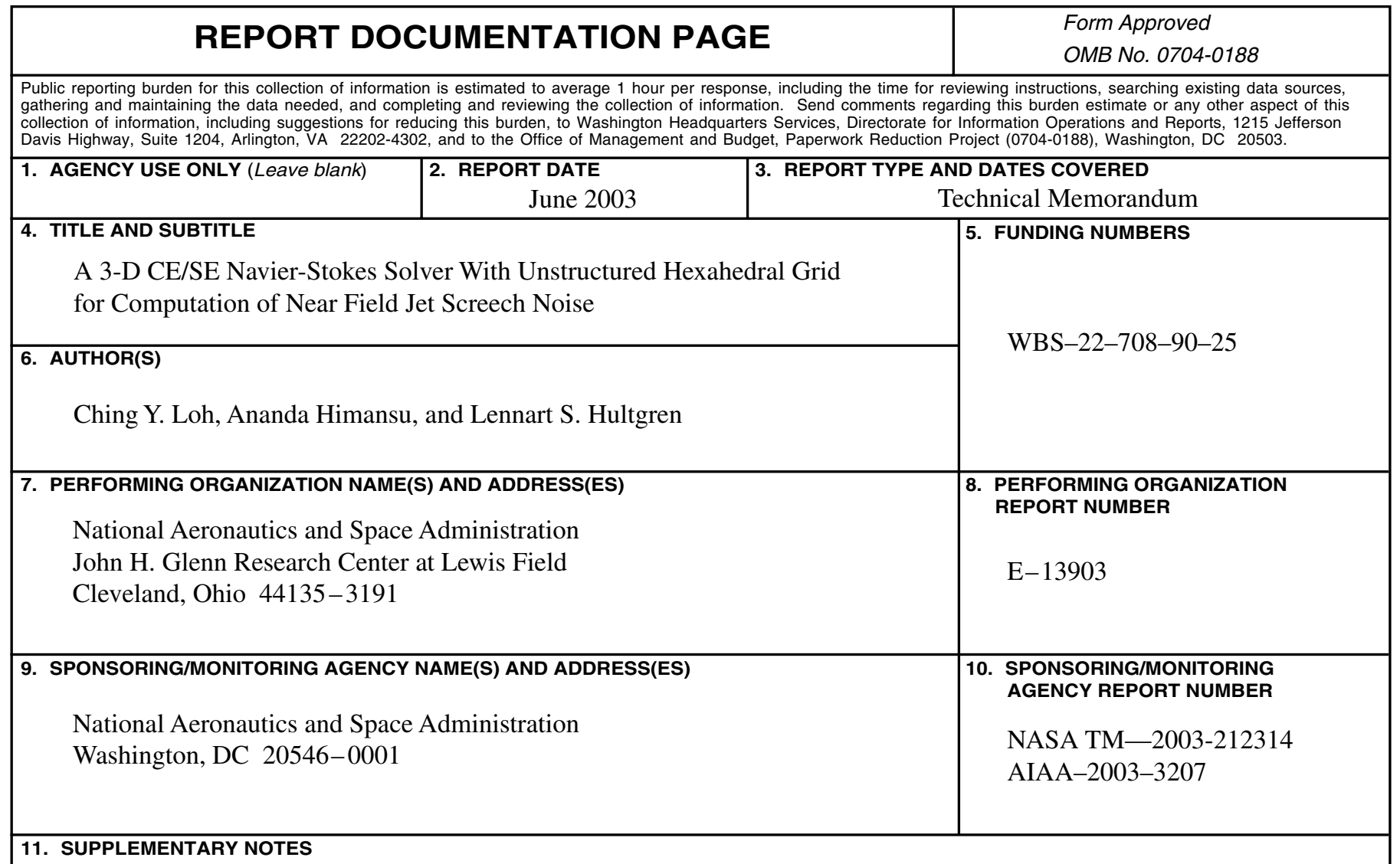

Prepared for the Nineth Aeroacoustics Conference and Exhibit cosponsored by the American Institute of Aeronautics and Astronautics and the Confederation of European Aerospace Societies, Hilton Head, South Carolina, May 12-14, 2003. Ching Y. Loh and Ananda Himansu, Taitech, Inc., Beaver Creek, Ohio 45430; Lennart S. Hultgren, NASA Glenn Research Center. Responsible person, Ching Y. Loh, organization code 5880, 216-433-3981.

\begin{tabular}{|l|l|l}
\hline 12a. & DISTRIBUTION/AVAILABILITY STATEMENT & 12b. DISTRIBUTION CODE \\
Unclassified - Unlimited & Distribution: Nonstandard & \\
Subject Categories: 02,71 , and $61 \quad$ & \\
Available electronically at http://gltrs.grc.nasa.gov & \\
This publication is available from the NASA Center for AeroSpace Information, 301-621-0390. & \\
\hline 13. ABSTRACT (Maximum 200 words)
\end{tabular}

A 3-D space-time CE/SE Navier-Stokes solver using an unstructured hexahedral grid is described and applied to a circular jet screech noise computation. The present numerical results for an underexpanded jet, corresponding to a fully expanded Mach number of 1.42, capture the dominant and nonaxisymmetric 'B' screech mode and are generally in good agreement with existing experiments.

\begin{tabular}{|c|c|c|c|}
\hline \multirow{2}{*}{\multicolumn{3}{|c|}{$\begin{array}{l}\text { 14. SUBJECT TERMS } \\
\text { CE/SE method; Unstructured hexahedral grid; Jet screech noise }\end{array}$}} & \multirow{3}{*}{$\begin{array}{l}\text { 15. NUMBER OF PAGES } \\
16 \\
\text { 16. PRICE CODE } \\
\text { 20. LIMITATION OF ABSTRACT }\end{array}$} \\
\hline & & & \\
\hline $\begin{array}{l}\text { 17. SECURITY CLASSIFICATION } \\
\text { OF REPORT } \\
\text { Unclassified }\end{array}$ & $\begin{array}{l}\text { 18. SECURITY CLASSIFICATION } \\
\text { OF THIS PAGE } \\
\text { Unclassified }\end{array}$ & $\begin{array}{l}\text { 19. SECURITY CLASSIFICATION } \\
\text { OF ABSTRACT } \\
\text { Unclassified }\end{array}$ & \\
\hline SN 7540-01-280-5500 & & & $\begin{array}{l}\text { ndard Form } 298 \text { (Rev. 2-89) } \\
\text { scribed by ANSI Std. Z39-18 } \\
-102\end{array}$ \\
\hline
\end{tabular}

